\title{
Parsaclisib Is a Next-Generation Phosphoinositide 3-Kinase $\delta$ Inhibitor with Reduced Hepatotoxicity and Potent Antitumor and Immunomodulatory Activities in Models of B-Cell Malignancy[
}

\author{
Niu Shin, Matthew Stubbs, Holly Koblish, Eddy W. Yue, Maxim Soloviev, Brent Douty, \\ Kathy He Wang, Qian Wang, Mingming Gao, Patricia Feldman, Gengjie Yang, Leslie Hall, \\ Michael Hansbury, Sybil O'Connor, Lynn Leffet, Robert Collins, Kamna Katiyar, Xin He, \\ Paul Waeltz, Paul Collier, Jin Lu, Yun-Long Li, Yanlong Li, Phillip C.C. Liu, ${ }^{1}$ Timothy Burn, \\ Maryanne Covington, Sharon Diamond, Dana Shuey, Alan Roberts, Swamy Yeleswaram, \\ Greg Hollis, Brian Metcalf, ${ }^{2}$ Wenqing Yao, Reid Huber, ${ }^{3}$ Andrew Combs, ${ }^{4}$ Robert Newton, \\ and Peggy Scherle ${ }^{4}$
}

Incyte Research Institute, Wilmington, Delaware

Received February 21, 2020; accepted April 20, 2020

\begin{abstract}
The clinical use of first-generation phosphoinositide 3-kinase $(\mathrm{PI} 3 \mathrm{~K}) \delta$ inhibitors in $\mathrm{B}$-cell malignancies is hampered by hepatotoxicity, requiring dose reduction, treatment interruption, and/or discontinuation of therapy. In addition, potential molecular mechanisms by which resistance to this class of drugs occurs have not been investigated. Parsaclisib (INCB050465) is a potent and selective next-generation PI3K $\delta$ inhibitor that differs in structure from first-generation $\mathrm{PI} 3 \mathrm{~K} \delta$ inhibitors and has shown encouraging anti-B-cell tumor activity and reduced hepatotoxicity in phase 1/2 clinical studies. Here, we present preclinical data demonstrating parsaclisib as a potent inhibitor of PI3K $\delta$ with over 1000 -fold selectivity against other class $1 \mathrm{PI} K \mathrm{~K}$ isozymes. Parsaclisib directly blocks PI3K signaling-mediated cell proliferation in B-cell lines in vitro and in vivo and indirectly controls tumor growth by lessening immunosuppression through regulatory T-cell inhibition in a syngeneic lymphoma model. Diffuse large B-cell lymphoma cell lines overexpressing MYC were insensitive to proliferation blockade via PI3K $\delta$ signaling inhibition by parsaclisib, but their proliferative activities were
\end{abstract}

reduced by suppression of $M Y C$ gene transcription. Molecular structure analysis of the first- and next-generation PI3K $\delta$ inhibitors combined with clinical observation suggests that hepatotoxicity seen with the first-generation inhibitors could result from a structure-related off-target effect. Parsaclisib is currently being evaluated in multiple phase 2 clinical trials as a therapy against various hematologic malignancies of B-cell origin (NCT03126019, NCT02998476, NCT03235544, NCT03144674, and NCT02018861).

\section{SIGNIFICANCE STATEMENT}

The preclinical properties described here provide the mechanism of action and support clinical investigations of parsaclisib as a therapy for B-cell malignancies. MYC overexpression was identified as a resistance mechanism to parsaclisib in DLBCL cells, which may be useful in guiding further translational studies for the selection of patients with DLBCL who might benefit from $\mathrm{PI} 3 \mathrm{~K} \delta$ inhibitor treatment in future trials. Hepatotoxicity associated with first-generation PI3K $\delta$ inhibitors may be an off-target effect of that class of compounds.

\section{Introduction}

The phosphoinositide 3-kinases (PI3Ks) regulate diverse biologic functions by generating lipid second messengers. PI3K $\delta$ is the main isozyme responsible for the activation of the PI3K

\footnotetext{
This work was supported by Incyte Corporation, Wilmington, Delaware. ${ }^{1}$ Current affiliation: Kymera Therapeutics, Cambridge, Massachusetts.

${ }^{2}$ Current affiliation: Private consultant, Moraga, California.

${ }^{3}$ Current affiliation: Third Rock Ventures, Boston, Massachusetts.

${ }^{4}$ Current affiliation: Prelude Therapeutics, Inc., Wilmington, Delaware. https://doi.org/10.1124/jpet.120.265538.
}

S This article has supplemental material available at jpet.aspetjournals.org. pathway in B-cell biology, functioning as a downstream mediator of the B-cell receptor (BCR), Toll-like receptors, and cytokine receptors (Puri and Gold, 2012). Because of the linkage of these signaling pathways to cancer biology, multiple drug discovery efforts to identify PI3K $\delta$ inhibitors for treating B-cell malignancies have been initiated (Greenwell et al., 2017). Inhibitors that are selective for PI3K $\delta$ versus other class I PI3K isoforms possess the advantage of avoiding toxicities that result from, for example, disruption of insulin signaling (PI3K $\alpha$ ) and spermatogenesis (PI3K $\beta$ ) (Hers, 2007; Ciraolo et al., 2010). Idelalisib, a firstgeneration PI3K $\delta$-selective inhibitor, is approved for the treatment of chronic and relapsed chronic lymphocytic leukemia (CLL),

ABBREVIATIONS: BCR, B-cell receptor; BET, bromodomain and extraterminal protein; CLL, chronic lymphocytic leukemia; DLBCL, diffuse large B-cell lymphoma; FL, follicular lymphoma; GLP, Good Laboratory Practice; MCL, mantle cell lymphoma; ORR, overall response rates; PI3K, phosphoinositide 3-kinase; TGl, tumor growth inhibition; Treg, regulatory T cell. 
small lymphocytic lymphoma, and follicular lymphoma (FL). Copanlisib, a pan-PI3K inhibitor with primary activity against $\alpha$ and $\delta$ isoforms, and duvelisib, a PI3K $\delta / \gamma$ dual inhibitor, were recently approved for FL and CLL/small lymphocytic lymphoma or FL, respectively. Several other PI3K $\delta$ inhibitors are at different stages of clinical development for B-cell-related diseases.

Clinical toxicities have hindered development of firstgeneration PI3K $\delta$-selective inhibitors, such as idelalisib and duvelisib. Hepatotoxicity is one of the most common adverse events, which in the case of idelalisib, has led to dose reduction and treatment discontinuation (Coutré et al., 2015). Therefore, there is an unmet need for a PI3K $\delta$ inhibitor with an improved safety profile, including minimal hepatotoxicity, as well as potent antitumor activity.

Idelalisib may cause hepatotoxicity by immune activation, since peripheral blood regulatory T cells (Tregs) were seen in patients experiencing hepatotoxicity while receiving idelalisib treatment (Lampson et al., 2016). Based on this hypothesis, hepatotoxicity would be predicted to be a class effect of PI3K $\delta$ inhibition. However, the possibility remained that idelalisib treatmentinduced hepatotoxicity could result from an off-target effect derived from its molecular structure. Thus, we initiated a discovery program for a next-generation PI3K $\delta$ inhibitor that differed in chemical structure from those of the first-generation compounds, including idelalisib. Parsaclisib (INCB050465) is a product of this discovery effort (Yue et al., 2019).

Parsaclisib is currently in phase 2 clinical trials as a therapy against various hematologic malignancies of B-cell origin. In a recent phase $1 / 2$ study (Forero-Torres et al., 2019), parsaclisib monotherapy exhibited high overall response rates (ORRs) in patients with relapsed or refractory mantle cell lymphoma (MCL) (ORR 67\%), FL (ORR 71\%), and marginal zone lymphoma (ORR 78\%), with lower responses observed in patients with relapsed or refractory diffuse large B-cell lymphoma (DLBCL; 30\%) and CLL (33\%). Most responses (93\%) occurred at first assessment ( 9 weeks). Importantly, about 10-fold reduced hepatotoxicity (measured in alanine aminotransferase/aspartate aminotransferase elevation $\geq$ grade 3 ) compared with first-generation PI3K $\delta$-selective inhibitors was reported in this study (Forero-Torres et al., 2019).

This study reports the mechanism of action of the antitumor activity of parsaclisib, which targets PI3K-mediated proliferative signaling in malignant B cells and lessens immunosuppression in the tumor microenvironment. These data suggest that $M Y C$ oncogene overexpression may be a mechanism by which resistance to PI3K $\delta$ inhibition by parsaclisib occurs in some DLBCL cell lines. In addition, based on structure analysis and clinical study results, a new hypothesis is proposed for the hepatotoxicity commonly seen in the first-generation PI3K $\delta$ inhibitors. These results not only provide mechanistic support for the ongoing clinical phase 2 studies of parsaclisib for treating different B-cell malignancies but may also be useful in guiding further translational studies for the selection of patients with DLBCL who might benefit from PI3K $\delta$ inhibitor treatment in future trials.

\section{Materials and Methods}

Chemicals, Antibodies, and Cell Lines. Parsaclisib and INCB054329 were synthesized at Incyte Corporation (Wilmington, DE). Supplier information for chemicals, antibodies, and cell lines used in the study is provided in the Supplemental Methods. All cell lines were maintained according to the instructions from suppliers.
Biochemical Assays. The detailed protocols for measuring biochemical activity of class 1 PI3K isozymes and 57 other kinases on the Incyte internal kinase panel have been described previously (Shin et al., 2018). The non-Good Laboratory Practice (GLP) General Side Effect PROFILE II screen at 0.1 and $1 \mu \mathrm{M}$ of parsaclisib in 70 in vitro binding or enzyme assays and the non-GLP PerkinElmer Discovery Services Kinase Assay screen of 192 kinases at $0.1 \mu \mathrm{M}$ of parsaclisib were evaluated by Caliper Life Sciences (Hanover, MD).

In Vitro Assays. The detailed protocol for Western blotting used to measure platelet-derived growth factor, lysophosphatidic acid, C5a, and anti-IgM-induced pAKT in NIH3T3, PC3, RAW264.7, and Ramos cells, respectively, was described previously (Shin et al., 2018). For measuring anti-IgM-induced pAKT in Ramos cells and C5a-induced pAKT in RAW264.7 cells, the cells were first serum starved in $0.2 \%$ FBS overnight, followed by treatment with parsaclisib for 2 hours, and then stimulated with anti-IgM $(4 \mu \mathrm{g} / \mathrm{ml})$ or C5a $(10 \mu \mathrm{g} /$ $\mathrm{ml}$ ) for 10 minutes at $37^{\circ} \mathrm{C}$. After fixation, the Ramos cells were stained with the anti-pAKT (Ser473)-Fluor 488 antibody and analyzed by fluorescence-activated cell sorting according to the antibody supplier's recommended protocol; the RAW264.7 cells were stained with an anti-pAKT (Ser473) antibody and analyzed with In-Cell Western analysis using the Odyssey CLx imaging system (Li-COR, Lincoln, NE) per the manufacturer's instruction. The constitutive level of pAKT in SKOV-3 and PC3-1 in the presence (2 hours) or absence of different concentrations of parsaclisib was measured by staining the cells with an anti-pAKT (Ser473) antibody followed by InCell Western analysis. The detailed procedures for purifying primary immune cells from different animal species and measuring proliferation of lymphoma cell lines and primary immune cells; differentiation of Th1, Th2, and Th17 cells; cytokine production from human memory $\mathrm{T}$ cells and natural killer cells; anti-FceR antibody-induced CD63 expression on basophils in the presence of human whole blood; and constitutive PI3K signaling in various lymphoma cell lines were described recently (Shin et al., 2018).

Animal Models. Non-GLP studies intended to characterize the pharmacology of parsaclisib were conducted in accordance with Incyte Corporation's Institutional Animal Care and Use Committee-approved animal use protocols. Animals were housed in barrier facilities fully accredited by the Association for Assessment and Accreditation of Laboratory Animal Care International. All procedures were conducted under the supervision of a veterinarian and in accordance with the US Public Health Service Policy on Humane Care and Use of Laboratory Animals.

For xenograft studies, $1 \times 10^{7}$ cells (WILL-2, Rec-1) were resuspended in Matrigel (BD Biosciences, Franklin Lakes, NJ) and injected subcutaneously into the dorsal flanks of severe combined immunodeficient mice (CB17/Icr-Prkdc scid/IcrIco Crl; Charles River Laboratories, Wilmington, MA). For the Pfeiffer model, subcutaneous tumors were grown to approximately $1000 \mathrm{~mm}^{3}$, harvested, cut into fragments of approximately $100 \mathrm{~mm}^{3}$, and then reimplanted subcutaneously into flanks of recipient mice. This results in more consistent tumor growth take-rates and kinetics. Subcutaneous tumors were allowed to grow until they reached approximately $200 \mathrm{~mm}^{3}$, and the mice were randomized into dosing groups. For tumor growth studies, tumors were measured at least twice weekly with electronic calipers. Tumor volumes were calculated using the following formula: volume = $\left(\right.$ length $\times$ width $\left.^{2}\right) / 2$, where width was the smaller dimension. Body weights were also monitored. For Western analyses of tumors, $1 \mathrm{~g}$ of tissue was homogenized with a handheld homogenizer in $15 \mathrm{ml}$ of Cell Lysis Buffer (Cell Signaling Technology, Danvers, MA) with Complete Mini EDTA-free protease inhibitor cocktail (Sigma, St. Louis, MO), including phenylmethanesulfonyl fluoride $(1 \mathrm{mM})$ and sodium orthovanadate $(2 \mathrm{mM})$. Statistical significance was determined by Student's $t$ test or two-way ANOVA using GraphPad Prism (La Jolla, CA) software.

Gene expression in tumor samples was assessed by RNAseq. Total RNA from frozen tissue samples was isolated using an RNeasy Mini Kit (Qiagen, Germantown, MD) in a QIACube (Qiagen, Hilden, Germany) according to the manufacturer's instructions. An optional 
DNase I digestion was performed. Sequencing was performed by BGI Americas (Cambridge, MA) on an Illumina Hi-seq4000 (San Diego, CA). Raw RNAseq fastq files were mapped to both human and mouse genome hg38. Clean human versions of gene transcripts were processed and quantified using OmicSoft ArrayStudio from Qiagen for further analyses. A general linear model for variant analysis was implemented in ArrayStudio. Gene Set Enrichment Analysis package was downloaded from the Broad Institute (Boston, MA).

For the syngeneic A20 lymphoma model, female BALB/c mice (5-9 weeks of age; Charles River Laboratories) were inoculated with A20 cells. A cell suspension in Hanks' balanced salt solution $\left(1 \times 10^{7}\right.$ cells $/ \mathrm{ml}$ ) was mixed 1:1 with Matrigel, and $200 \mu \mathrm{l}$ was injected subcutaneously into the flank of each mouse. The treatment of the tumor-bearing mice started 7-24 days after tumor inoculation for efficacy studies. Mice were sorted to obtain approximately equivalent mean tumor volumes in each group. The starting mean tumor volume in efficacy studies for all groups ranged from 158 to $249 \mathrm{~mm}^{3}$, and groups consisted of eight or nine animals. Parsaclisib was administered by oral gavage twice daily for 7-19 days (suspension in $5 \%$ dimethylacetamide with $0.5 \%$ w $/ \mathrm{v}$ methylcellulose). For the statistical significance analysis, unpaired $t$ test with Mann-Whitney post-test of tumor dimensions was performed with GraphPad Prism v.7.

To evaluate pharmacodynamics of parsaclisib in the A20 model, mice were euthanized after 7 days of dosing, and their tumors and spleens were excised ( 2 hours after final dose) and collected into Roswell Park Memorial Institute media (Gibco, Gaithersburg, MD). Tumors were coarsely minced and then processed to single-cell suspension by GentleMacs and Mouse Tumor Dissociation enzyme mix (Miltenyi, Sunnyvale, CA) per the manufacturer's protocol.
Spleens were diced and passed through Falcon mesh cell strainers (Fisher Scientific, Pittsburgh, PA), and red blood cells were lysed with Pharm Lyse (BD Biosciences) and then washed in PBS. All single-cell suspensions were resuspended in staining buffer (BD Biosciences) at $1 \times 10^{8} \mathrm{cells} / \mathrm{ml}$. The cells were treated with Fc block (BD Biosciences) for 5 minutes to reduce nonspecific bindings of antibodies to Fc receptors on cells and then stained with antibodies as described in the text, followed by fluorescence-activated cell sorting analysis.

Parsaclisib was evaluated in GLP 28-day oral gavage toxicology studies in Sprague-Dawley rats (15 per sex per group) at dosages of 0, 10, 30 , or $100 \mathrm{mg} / \mathrm{kg}$ per day and beagle dogs at $0,1,3$, or $15 \mathrm{mg} / \mathrm{kg}$ per day, which included a 4-week recovery period, according to a standard protocol.

\section{Results}

Parsaclisib Is a Highly Potent and Selective PI3K $\delta$ Inhibitor. Parsaclisib ((R)-4(3-((S)-1-(4-amino-3-methyl- $1 H$ pyrazolo[3,4-day] pyrimidin-1-yl)ethyl)-5-chloro-2-ethoxy-6fluorophenyl)pyrrolidin-2-one) (Fig. 1A) resulted from our efforts to identify a potent, selective, and structurally diverse PI3K $\delta$ inhibitor (Yue et al., 2019). In biochemical assays, parsaclisib potently inhibited PI3K $\delta$ activity $\left(\mathrm{IC}_{50}=1 \mathrm{nM}\right)$ and was at least 1000 -fold more selective for PI3K $\delta$ compared with the other three members of the class 1 PI3Ks $(\mathrm{PI} 3 \mathrm{~K} \alpha, \mathrm{PI} 3 \mathrm{~K} \beta$, and PI3K $\gamma$ ) (Fig. 1B). The selectivity of parsaclisib was further evaluated using a general side effect profile screen, conducted by Caliper Life Sciences (Hopkinton, MA). The results showed no interactions with 70 different channels and transporters
A $\quad$ B<smiles>CCOc1c([C@@H](C)n2nc(C)c3c(N)ncnc32)cc(Cl)c(F)c1C1CNC(=O)C1</smiles>

\begin{tabular}{|c|c|c|c|c|}
\hline & $\begin{array}{c}\text { PI3Ka } \\
\left(\mathrm{II}_{50} \pm \text { S.D., nM) }\right.\end{array}$ & $\begin{array}{c}\text { PI3K } \beta \\
\left(\mathrm{IC}_{50} \pm \text { S.D., nM) }\right.\end{array}$ & $\begin{array}{c}\text { PI3Kס } \\
\left(\mathrm{IC}_{50} \pm \text { S.D., nM) }\right.\end{array}$ & $\begin{array}{c}\text { PISKY } \\
\left(I \mathrm{C}_{50} \pm \text { S.D., nM) }\right.\end{array}$ \\
\hline & $>10,000$ & $>10,000$ & $1 \pm 1$ & $>10,000$ \\
\hline & $7736 \pm 2031$ & $690 \pm 188$ & $16 \pm 6$ & $3086 \pm 313$ \\
\hline
\end{tabular}

C

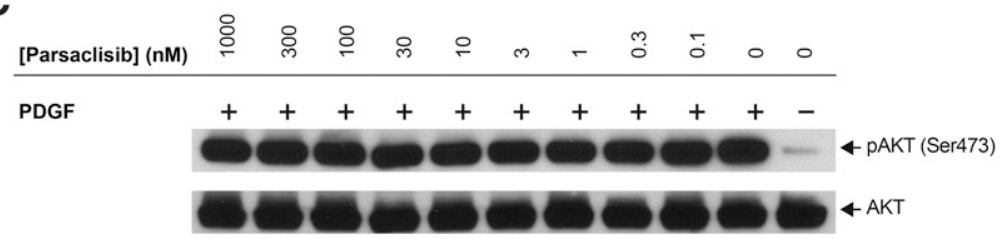

LPA

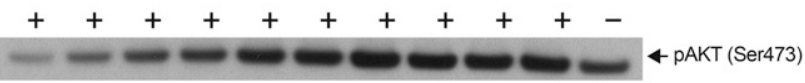

Anti-IgM
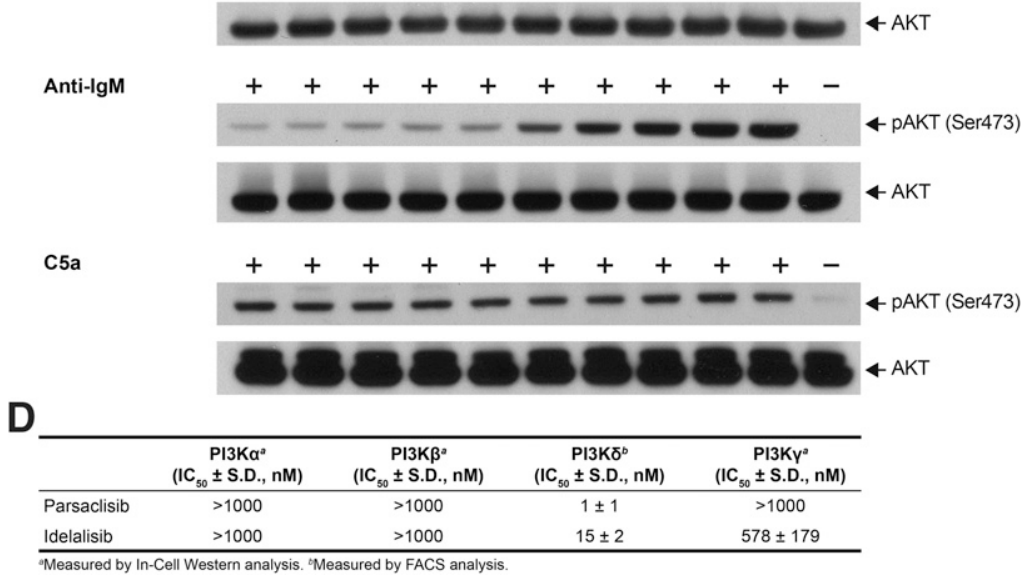

Fig. 1. Parsaclisib chemical structure and activity against class 1 PI3K. (A) Chemical structure of parsaclisib $[((\mathrm{R})$ 4(3-((S)-1-(4-amino-3-methyl-1 $H$-pyrazolo [3,4-day]pyrimidin-1-yl)ethyl)-5-chloro-2ethoxy-6-fluorophenyl)pyrrolidin-2-one)]. (B) Enzyme-inhibitory activity of parsaclisib at $1 \mathrm{mM}$ concentration of ATP against different members of the human class 1 PI3K family $(n \geq 3)$. Idelalisib was tested in the same assays for comparison purposes. (C) Parsaclisib activity against PI3K $\alpha-$, PI3K $\beta-$, PI3K $\delta$-, and PI3K $\gamma$ mediated signaling in cells by Western blotting. pAKT (Ser473) was induced by platelet-derived growth factor (PDGF), lysophosphatidic acid (LPA), anti-IgM, and C5a in NIH3T3, PC-3, Ramos, and RAW264.7 cells, respectively, for PI3K $\alpha$-, $\mathrm{PI} 3 \mathrm{~K} \beta$-, PI3K $\delta$-, and PI3K $\gamma$-mediated signaling. The levels of pAKT (Ser473) and total AKT were measured by Western blotting. The data are representative of two independent experiments. (D) The activities of parsaclisib and idelalisib against $\mathrm{PI} 3 \mathrm{~K} \alpha-$, PI3K $\beta$-, PI3K $\delta$-, and PI3K $\gamma$-mediated signaling measured directly in cells. Constitutive levels of pAKT (Ser473) in SKOV-3 and PC-3 cells measured by In-Cell Western imaging were used to represent $\mathrm{PI} 3 \mathrm{~K} \alpha$ and $\mathrm{PI} 3 \mathrm{~K} \beta$ activities, respectively. PI3K $\gamma$ activity in RAW264.7 cells was measured by the level of pAKT (Ser473) induced by C5a and detected with In-Cell Western imaging. PI3K $\delta$ activity in Ramos cells was measured by the level of pAKT (Ser473) induced by anti-IgM and detected with fluorescence-activated cell sorting (FACS) analysis $(n \geq 3)$. Idelalisib was tested in the same assays for comparison purposes. 
and demonstrated little or no inhibition ( $\leq 15 \%$ inhibition) of 192 kinases screened (data not shown).

In a cell-based assay, parsaclisib was active in suppressing PI3K $\delta$ signaling as measured by its inhibitory effect on antiIgM-induced pAKT (Ser473) in the Ramos Burkitt's lymphoma cell line with an average $\mathrm{IC}_{50}$ value of approximately 1 nM (Fig. 1, C and D). Consistent with the enzyme assay results, parsaclisib was much less active against $\mathrm{PI} 3 \mathrm{~K} \alpha$ - and PI3K $\gamma$-mediated signaling. $\mathrm{IC}_{50}$ values were $>1000 \mathrm{nM}$ for the inhibition of PI3K $\alpha$ by parsaclisib, assessed based on plateletderived growth factor-induced pAKT (Ser473) in NIH3T3 fibroblasts and constitutive pAKT (Ser473) in the SKOV-3 cells (Whyte and Holbeck, 2006; Lannutti et al., 2011), and $>1000 \mathrm{nM}$ for the inhibition of PI3K $\gamma$ in C5a-induced pAKT (Ser473) in the RAW264.7 mouse macrophage cells (Evans et al., 2016) (Fig. 1, C and D). The inhibitory activity of parsaclisib against $\mathrm{PI} 3 \mathrm{~K} \beta$ in cells varied depending on the assay conditions used: the $\mathrm{IC}_{50}$ was $>1000 \mathrm{nM}$, based on measurements of constitutive pAKT (Ser473) in the In-Cell Western assay using the PC3 cell line (Wee et al., 2008); however, the $\mathrm{IC}_{50}$ was lower (approximately $30 \mathrm{nM}$ ), based on measurements of lysophosphatidic acid-induced pAKT (Ser473) in Western blots using PC3 cells (Lannutti et al., 2011), thus demonstrating $\geq 30$-fold selectivity of PI3K $\delta$ over PI3K $\beta$ (Fig. 1, C and D). Idelalisib, evaluated in the same biochemical and cellular assays, demonstrated less selectivity for PI3K $\delta$ compared with parsaclisib (Fig. 1, B and D).

Parsaclisib Inhibits PI3Ko Functions in Primary Immune Cells. PI3K $\delta$ is critical for B-cell survival and proliferation by functioning downstream of prosurvival receptors such as the BCR, B-cell activating factor receptor, and the interleukin-4 receptor, as well as costimulatory receptors such as CD40 and Toll-like receptors (Puri and Gold, 2012). Parsaclisib inhibited the proliferation of human, dog, rat, and mouse primary $B$ cells after activation of these receptors, with $\mathrm{IC}_{50}$ values ranging from 0.2 to $1.7 \mathrm{nM}$ (Supplemental Table 1). PI3K $\delta$ is also known to be critical for differentiation of Th1, Th2, and Th17 cells (Okkenhaug et al., 2006; HaylockJacobs et al., 2011; Kurebayashi et al., 2012; Roller et al., 2012; Steinbach et al., 2014) and cytokine production in memory T cells (Soond et al., 2010). Consistent with this, parsaclisib demonstrated potent inhibitory activity against these PI3K $\delta$ mediated functions in $\mathrm{T}$ cells $\left(\mathrm{IC}_{50}\right.$ values ranging from 0.2 to $1.5 \mathrm{nM}$ ) (Supplemental Table 1). Parsaclisib was further evaluated for its potency in inhibiting anti-FceR antibodyinduced CD63 expression on basophils in human whole blood as previously described (Lannutti et al., 2011), demonstrating a mean $\mathrm{IC}_{50}$ of approximately $2 \mathrm{nM}$ (Supplemental Table 1).

Ablation of PI3K $\delta$ catalytic function using a p $110 \delta^{\mathrm{D} 910 \mathrm{~A} / \mathrm{D} 910 \mathrm{~A}}$ mouse model does not impact on CD3-induced T-cell proliferation in the presence of CD28 costimulation, suggesting that CD28 may use PI3K $\delta$-independent pathways to enhance T-cell receptor signaling in $\mathrm{p} 110 \delta^{\mathrm{D} 910 \mathrm{~A} / \mathrm{D} 910 \mathrm{~A}}$-expressing $\mathrm{T}$ cells (Okkenhaug et al., 2002, 2006). Consistent with these findings, we observed that parsaclisib did not inhibit anti-CD3 or antiCD3 plus anti-CD28 antibody-induced proliferation of human T cells $\left(\mathrm{IC}_{50}>1000 \mathrm{nM}\right)$ and only modestly inhibited anti-CD28 antibody-induced T-cell proliferation $\left(\mathrm{IC}_{50}=96 \mathrm{nM}\right)$ (Supplemental Table 1). Parsaclisib also did not inhibit interleukin-2-induced human natural killer cell proliferation but was active in suppressing differentiation of naïve human T cells into a Th17, Th1, or Th2 phenotype and inhibiting functions of human memory $\mathrm{T}$ cells measured by cytokine production (Supplemental Table 1).

Parsaclisib Inhibits Proliferation of MCL and DLBCL Cell Lines In Vitro and In Vivo. The antiproliferative effect of parsaclisib on B-cell lymphoma cell lines was assessed using the $\left[{ }^{3} \mathrm{H}\right]$ thymidine uptake assay. In the four MCL cell lines tested (Jeko-1, Mino, Rec-1, and JVM2), treatment with parsaclisib resulted in a maximal inhibition of $70 \%-90 \%$, with $\mathrm{IC}_{50}$ values $\leq 10 \mathrm{nM}$ (Fig. 2A). Signaling studies in these cells showed that parsaclisib reduced the levels of constitutive pAKT (Ser473) and pFOXO1 with similar potencies (Fig. 2B). The level of FOXO1 phosphorylation was investigated, as it is a signaling event known to be downstream of AKT activation by PI3K in the regulation of cell proliferation (Fabre et al., 2005).

Among the seven DLBCL cell lines (all germinal center B-cell-like subtype) that were evaluated for their sensitivity to parsaclisib in the proliferation assay, four (Pfeiffer, SUDHL-5, SU-DHL-6, and WSU-NHL) were highly sensitive, with $\mathrm{IC}_{50}$ values from 2 to $8 \mathrm{nM}$ (Fig. $2 \mathrm{C}$ ). The remaining three cell lines (SU-DHL-4, SU-DHL-8, and WILL-2) showed no sensitivity to parsaclisib, although the levels of pAKT (Ser473) and pFOXO1 in all seven DLBCL cell lines were similarly reduced by compound treatment (Fig. 2D).

To determine whether the broad antiproliferative effects seen with parsaclisib in vitro could also be observed in vivo, we used Pfeiffer (DLBCL) and Rec-1 (MCL) subcutaneous mouse xenograft models. Using pAKT (Ser473) as a pharmacodynamic marker of PI3K $\delta$ in Pfeiffer xenograft tumors, parsaclisib inhibited pAKT (Ser473) within 4 hours of a single oral administration, consistent with in vivo parsaclisib activity (Supplemental Fig. 1A). Based on the pharmacokinetic properties of parsaclisib in mice (Supplemental Fig. 1B), and the effect on pAKT levels in vivo, a dose range of $0.1-10 \mathrm{mg} / \mathrm{kg}$ twice daily for efficacy studies was chosen to maintain adequate levels of parsaclisib over a 24-hour period. Parsaclisib slowed Pfeiffer xenograft tumor growth in a dosedependent manner, with significant tumor growth inhibition (TGI) occurring with 1 and $10 \mathrm{mg} / \mathrm{kg}$ twice daily dose levels (Fig. 3A). In each study, parsaclisib was well tolerated as monitored by nonsignificant changes in body weight with treatment. Likewise, parsaclisib significantly slowed Rec-1 xenograft tumor growth at each dose used (Fig. 3B). Parsaclisib also showed additive effects with bendamustine, a cytotoxic alkylating chemotherapeutic agent for treating indolent B-cell malignancies, in the Pfeiffer and Rec-1 models (Fig. 3, C and D), which provides translational evidence to support future clinical study of parsaclisib plus bendamustine in patients with B-cell lymphoma.

Bromodomain and Extraterminal Protein Inhibition Overcomes Insensitivity to Parsaclisib-Mediated Growth Inhibition in DLBCL With MYC Dysregulation. The insensitivity of the SU-DHL-4, SU-DHL-8, and WILL-2 cell lines to parsaclisib was in stark contrast to the sensitivity of other lymphoma lines tested. Parsaclisib reduced pAKT (Ser473) and pFOXO1 levels in SU-DHL-4, SU-DHL-8, and WILL-2 cells yet did not inhibit their proliferation. One possible reason for this insensitivity to $\mathrm{PI} 3 \mathrm{~K} \delta$ inhibition is aberrant expression of MYC as an overriding proliferative signal. Several reports have shown that $M Y C$ dysregulation may be an underlying mechanism for resistance to PI3K inhibition (Ilic et al., 2011; Liu et al., 2011). However, the role 
A

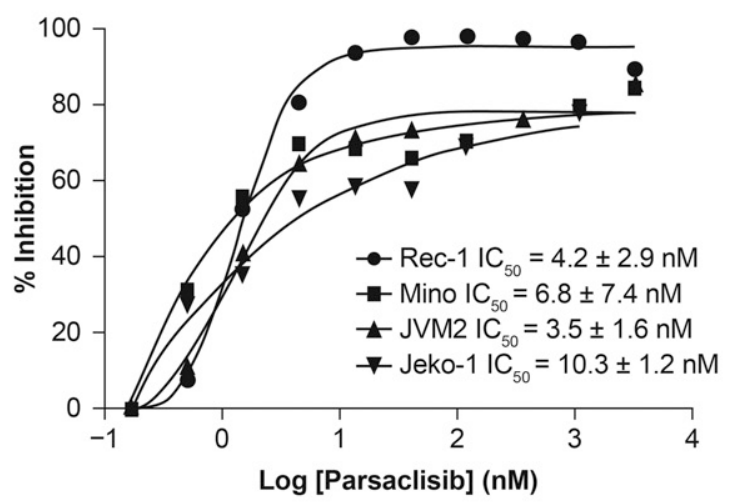

C

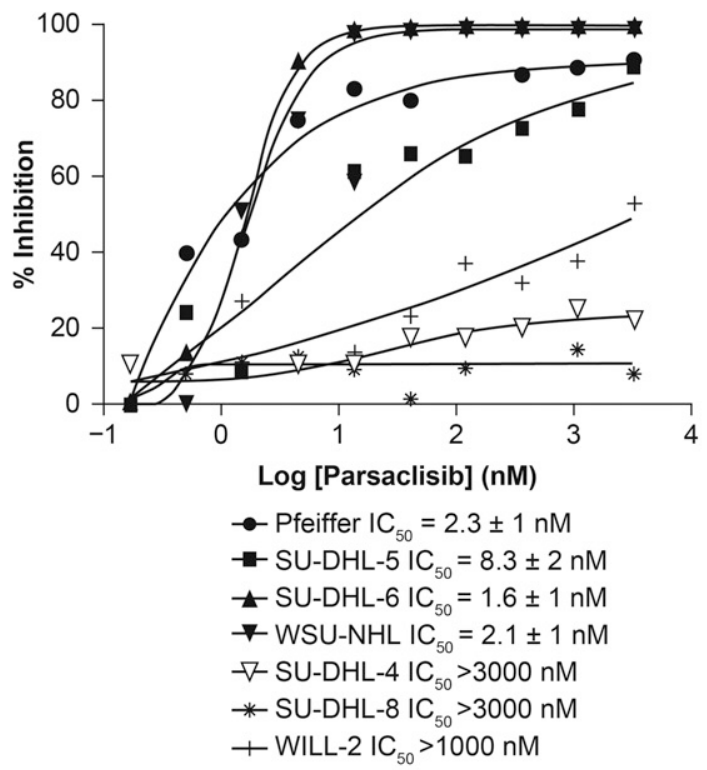

B

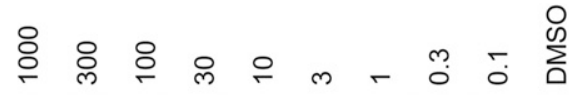

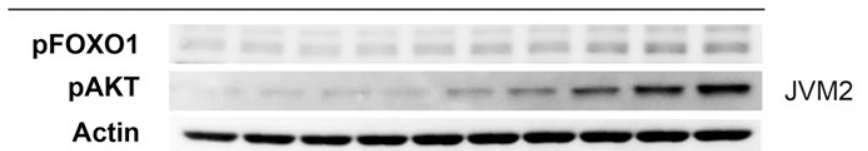

D

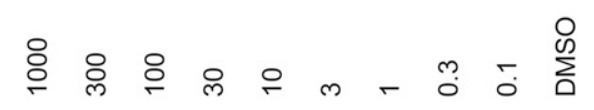

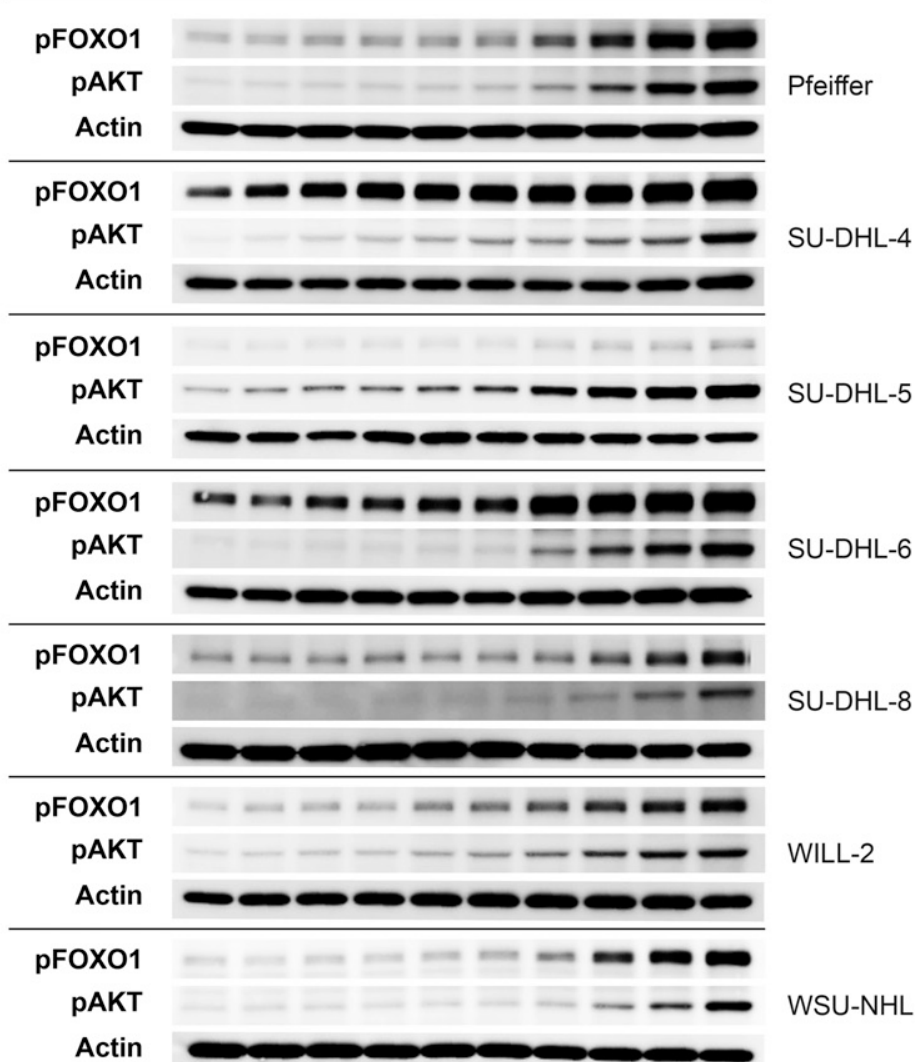

Fig. 2. Parsaclisib inhibits proliferation and constitutive PI3K signaling in MCL and DLBCL cell lines in vitro. (A) Jeko-1, Mino, JVM2, and Rec-1 cells (MCL) were cultured in growth medium for 4 days in the presence of different concentrations of parsaclisib and then pulsed with $\left[{ }^{3} \mathrm{H}\right]$ thymidine overnight. Incorporated radioactivity was counted as measurement of cell growth. Each curve is a representative and value avg. of multiple individual experiments $(n=5$ for Rec-1, $n=5$ for Mino, $n=5$ for JVM2, and $n=3$ for Jeko-1). (B) The MCL cell lines in growth medium were treated with different concentrations of parsaclisib for 2 hours. The levels of pAKT (Ser473) and pFOXO1 (T24) in the total cell lysates were then measured by Western blotting. Actin levels in the cell lysates was used as loading control. These data are representative of two independent experiments. (C) Pfeiffer, SUDHL-5, SU-DHL-6, WSU-NHL, SU-DHL-4, SU-DHL-8, and WILL-2 cells (DLBCL, all germinal center B-cell-like subtype) were cultured in growth medium for 4 days in the presence of different concentrations of parsaclisib and then pulsed with $\left[{ }^{3} \mathrm{H}\right]$ thymidine overnight. Incorporated radioactivity was counted as measurement of cell growth. Each curve is a representative and value avg. of multiple individual experiments $(n=9$ for Pfeiffer, $n=4$ for SU-DHL-5, $n=6$ for SU-DHL-6, $n=4$ for WSU-NHL, $n=3$ for SU-DHL-4, $n=3$ for SU-DHL-8, and $n=3$ for WILL-2). (D) The DLBCL cells in growth medium were treated with different concentrations of parsaclisib for 2 hours. The levels of pAKT (Ser473) and pFOXO1 (T24) in the total cell lysates were then measured by Western blotting. Actin levels in the cell lysates were used as loading control. The data are representative of two independent experiments. 
A

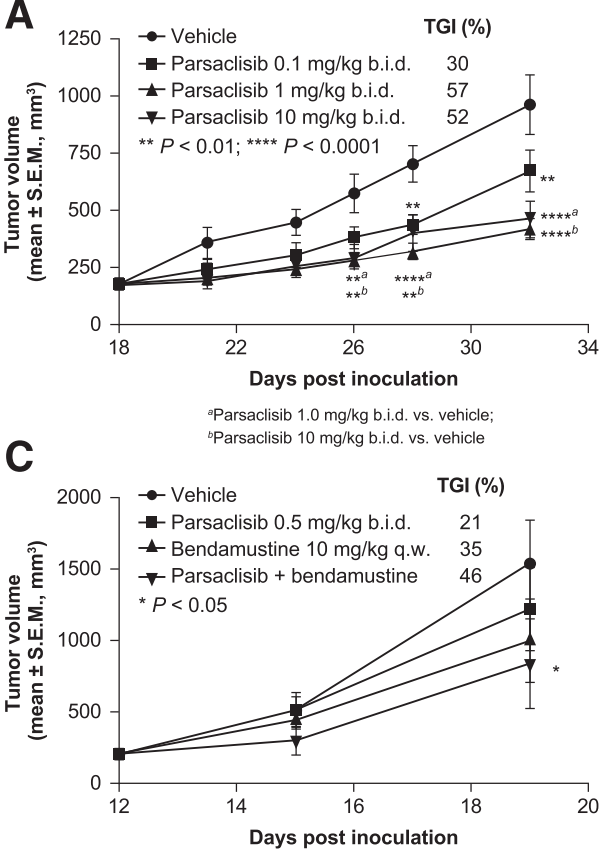

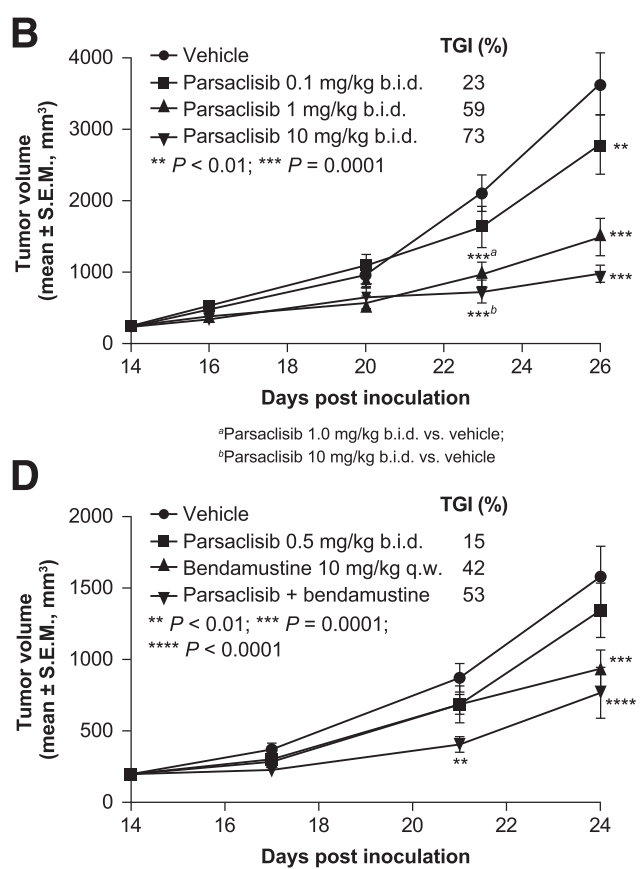

Fig. 3. Parsaclisib is active as a single agent and in combination with standardof-care chemotherapy against tumor growth in the Pfeiffer and Rec-1 models for DLBCL and MCL. Efficacy of parsaclisib in the Pfeiffer (A) and Rec-1 (B) subcutaneous xenograft models. Efficacy of parsaclisib in combination with bendamustine in the Pfeiffer (C) and Rec-1 (D) subcutaneous xenograft models. The difference in percentage TGI was analyzed by $t$ test. b.i.d., twice daily; q.w., once weekly. of $M Y C$ in the mechanism of resistance to $\mathrm{PI} 3 \mathrm{~K} \delta$-specific inhibition has not yet been determined. Both SU-DHL-8 and WILL-2 cells carry the $\mathrm{t}(8 ; 22)$ chromosome translocation, resulting in $\operatorname{Ig} L-M Y C$ rearrangement and presumably increased $M Y C$ transcription. Although SU-DHL-4 cells have no $M Y C$-associated translocation, they do express a high level of MYC protein (Schrader et al., 2012). Using Western blotting, the expression of MYC was barely detectable in the four parsaclisib-sensitive DLBCL cell lines (Pfeiffer, SU-DHL5, SU-DHL-6, and WSU-NHL) compared with strong expression in the three parsaclisib-insensitive DLBCL cell lines (WILL-2, SU-DHL-4, and SU-DHL-8) (Fig. 4A).

The striking difference in MYC levels between parsaclisibsensitive and -insensitive cell lines suggests that modulation of MYC levels may result in heightened sensitivity to PI3K $\delta$ inhibition. Bromodomain and extraterminal domain (BET) family of proteins are epigenetic modulators of gene expression that bind selectively to acetylated histone lysine residues and have been shown to stimulate growth of malignant B cells by promoting MYC expression (Delmore et al., 2011; Boi et al., 2015). We tested SU-DHL-4, SU-DHL-8, and WILL-2 cells with the BET inhibitor INCB054329 (Stubbs et al., 2019). All three parsaclisib-insensitive DLBCL cell lines were sensitive to BET inhibition by INCB054329 in an in vitro proliferation assay (Fig. 4B). No synergistic or additive effects were observed between parsaclisib and INCB054329 in the same in vitro assay (data not shown). Concomitantly, the expression of MYC was reduced by INCB054329 in a dose-dependent manner (Fig. 4C). The potency of the inhibitory effect of INCB054329 on MYC expression appeared to be similar to that for the inhibitory effect on cell proliferation.

The ability of parsaclisib-insensitive cell lines to respond to BET inhibition with INCB054329 in vivo was tested in WILL-2 subcutaneous tumors. The effects of parsaclisib or INCB054329 on TGI were not statistically different compared with the vehicle control. However, the TGI resulting from the combination of both compounds at the same doses differed significantly from each single-agent group and from the vehicle control. Importantly, all doses were well tolerated as monitored by nonsignificant changes in body weight with treatment. These data suggest that suppression of PI3K $\delta$ with parsaclisib may enhance the sensitivity of lymphomas with $M Y C$ overexpression to BET inhibition with INCB054329 in vivo (Fig. 4D).

To understand the potential mechanism(s) behind this apparent synergy between $\mathrm{PI} 3 \mathrm{~K} \delta$ and BET inhibition in the WILL-2 model, gene expression profiles in the tumors were analyzed by RNAseq. Based on a general linear model and hierarchical clustering of 5654 differentially expressed genes, the combination treatment of parsaclisib and INCB054329 resulted in a distinct gene expression pattern when compared with either parsaclisib or INCB054329 alone (Fig. 4E). In particular, gene set enrichment analyses showed strong suppression of MYC-associated genes (Delmore et al., 2011; Lampson et al., 2016) as well as an enrichment of a p53 pathway signature (Fig. 4F), indicating that simultaneous $\mathrm{BET}$ and $\mathrm{PI} 3 \mathrm{~K} \delta$ inhibition can inhibit cell growth via several mechanisms in PI3K $\delta$ inhibitor-insensitive cells. Together, high expression of both MYC and p53 correlate with poor prognosis in DLBCL (Richardson et al., 2019). Importantly, of the 3363 genes differentially expressed after combination treatment of parsaclisib and INCB054329, 2389 were uniquely regulated when compared with the single-agent groups (Supplemental Table 2). Genes showing significantly decreased expression with combination treatment versus vehicle included CCR6, CCR2, CCR5, CCR1, CXCR2, $C C L 3 L 3$, and CCL1 $(P<0.05)$. This suggests that tumor cell networks mediated by chemokine and chemokine receptors could also be affected.

Parsaclisib Functions As an Immunomodulator and Inhibits Tumor Growth in the A20 Syngeneic Mouse Model for Lymphoma. In addition to its direct antiproliferative activity through inhibition of intracellular signaling in malignant $B$ cells and effect on chemokine and chemokine receptor networks, we hypothesized that $\mathrm{PI} 3 \mathrm{~K} \delta$ inhibition can potentially suppress tumor growth through modulation of 
A

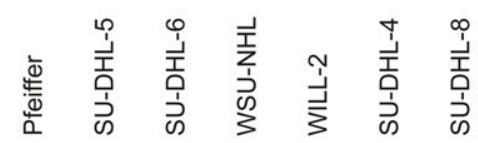

MYC

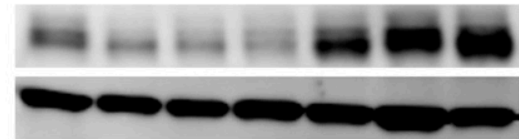

B

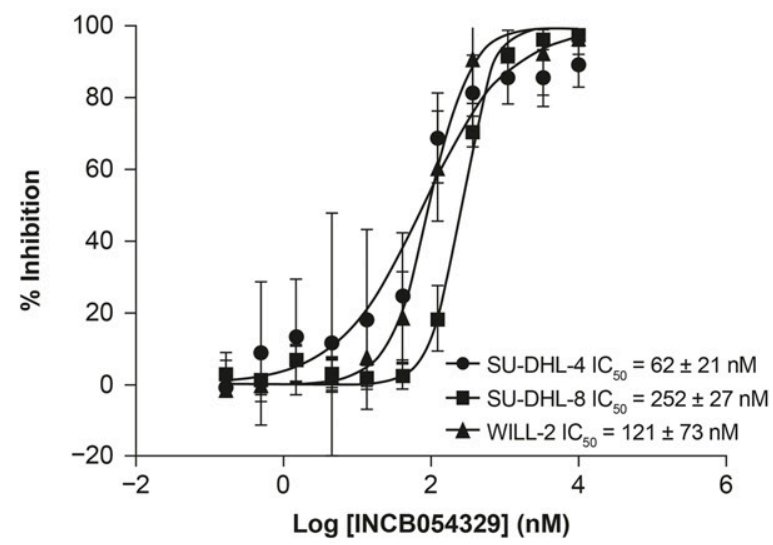

C

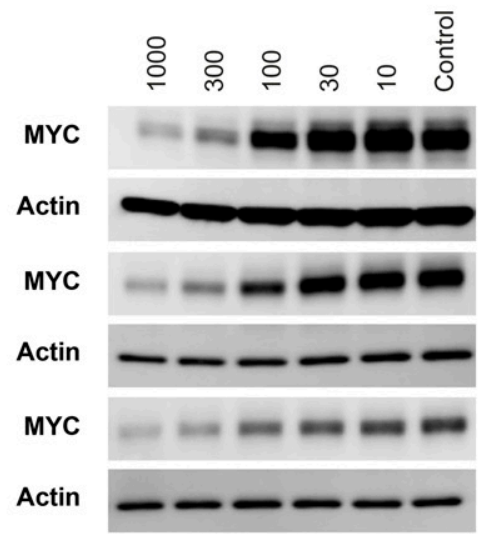

SU-DHL-4

E

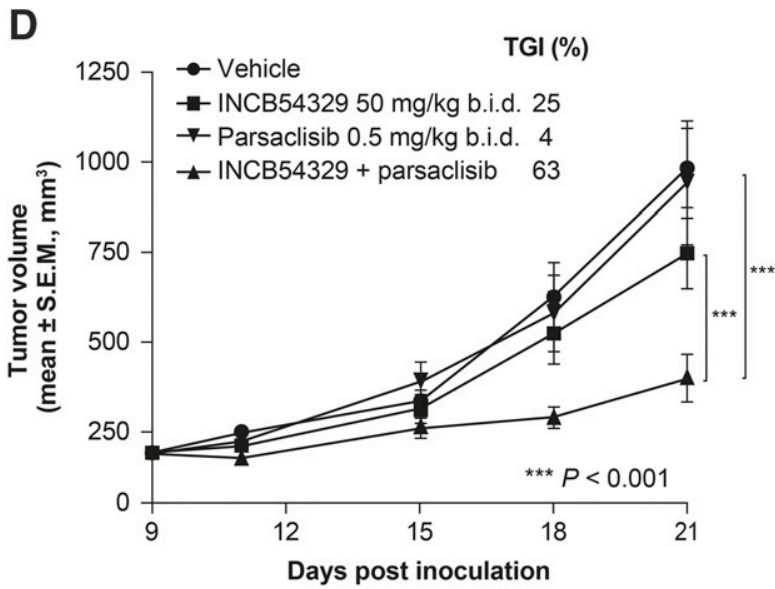

$\mathbf{F}$

Enrichment plot: HALLMARK_P53_PATHWAY

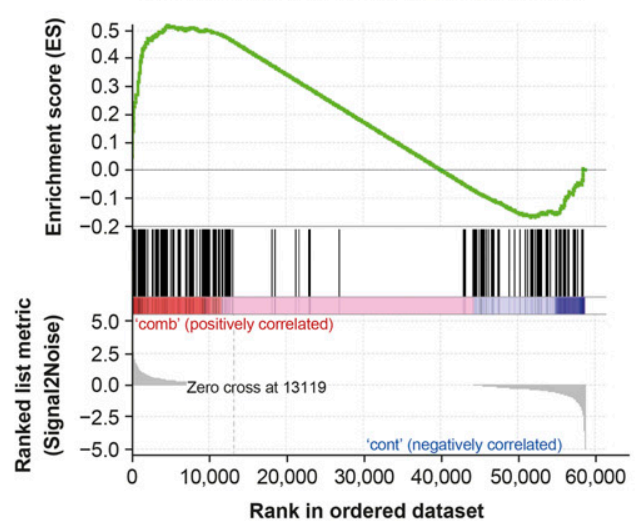

- Enrichment profile — Hits

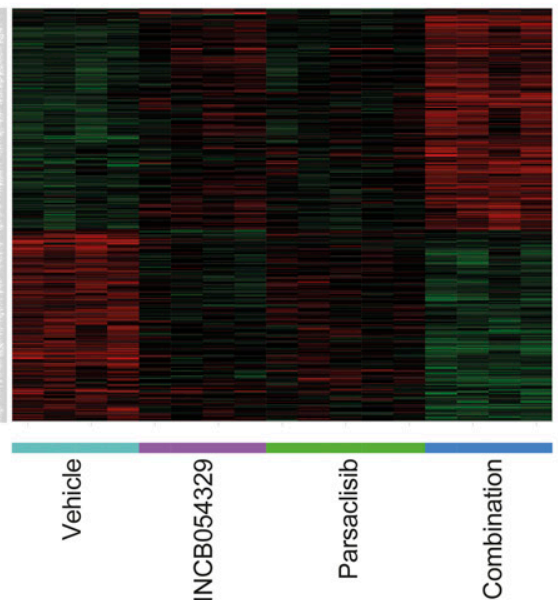

Enrichment plot: HALLMARK_MYC_TARGETS_V2

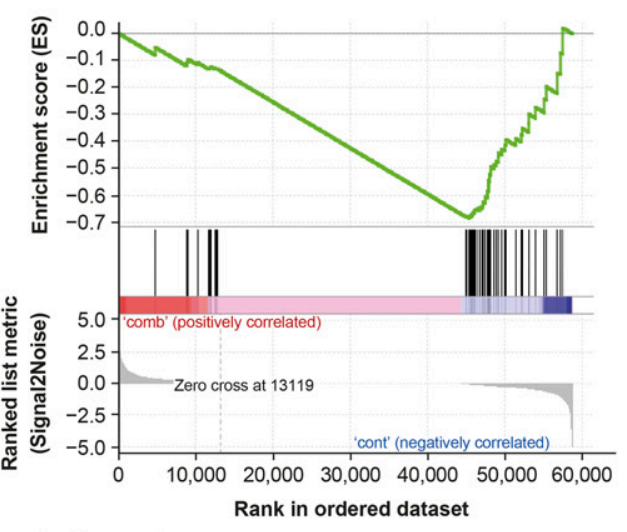

Fig. 4. Insensitivity to parsaclisib in DLBCL cells with high MYC expression can be overcome by BET inhibition. (A) Comparison of MYC expression in four parsaclisib-sensitive DLBCL cell lines (Pfeiffer, SU-DHL-5, SU-DHL-6, and WSU-NHL) and three parsaclisib-insensitive DLBCL cell lines (WILL2, SU-DHL-4, and SU-DHL-8). (B) $\left[{ }^{3} \mathrm{H}\right]$ thymidine uptake proliferation assays were performed on the three parsaclisib-insensitive DLBCL cell lines (SUDHL-4, SU-DHL-8, and WILL-2) treated with the BET inhibitor INCB054329. The data represent an avg. of three independent experiments. (C) 
regulatory and cytotoxic $\mathrm{T}$ cells within the B-cell lymphoma microenvironment. $\mathrm{PI} 3 \mathrm{~K} \delta$ activity has been shown to be critical for the immunosuppressive function of Tregs (Patton et al., 2006). Inhibition of PI3K $\delta$ has also been suggested to delay terminal differentiation of $\mathrm{CD}^{+} \mathrm{T}$ cells and maintain memory phenotype, cytokine, and granzyme B production (Abu Eid et al., 2017). A syngeneic A20 lymphoma model was used to assess the effect of parsaclisib on tumor growth through modulation of immune cells in a B-cell lymphoma microenvironment. A20 cells are relatively insensitive to parsaclisib in an in vitro proliferation assay $\left(\mathrm{IC}_{50}>3000\right.$ $\mathrm{nM})$. However, treatment of BALB/c mice bearing the A20 murine lymphoma cells with parsaclisib resulted in significant TGI (Fig. 5A).

In support of the observed antitumor effect of parsaclisib in the A20 model, treatment with parsaclisib reduced the percentage of Tregs $\left(\mathrm{CD} 4^{+} \mathrm{CD} 25^{+} \mathrm{FOXP} 3^{+}\right)$in tumors and spleens (Fig. 5B; Supplemental Fig. 2A). In line with the decrease in Tregs, the ratio of $\mathrm{CD}^{+}$and $\mathrm{CD}^{+} \mathrm{T}$ cells to Tregs in spleens and tumors was increased by treatment with parsaclisib (Fig. 5, C and D). Because PI3K $\delta$ has been implicated in T-cell memory phenotype (Abu Eid et al., 2017), the effect of parsaclisib on this subset of T cells in the A20 model was analyzed. Parsaclisib treatment resulted in a decrease in the number of $\mathrm{CD} 4^{+} \mathrm{CD} 44^{\text {high }}$ and $\mathrm{CD}^{+} \mathrm{CD} 44^{\text {high }} \mathrm{T}$ cells in both spleens and tumors (Fig. 5, E and F; Supplemental Fig. 2B). Further analysis of the CD44 ${ }^{\text {high }}$ T-cell population, which is considered to be a marker for the activated/memory $\mathrm{T}$ cells, showed that $\mathrm{PI} 3 \mathrm{~K} \delta$ inhibition by parsaclisib increased the percentage of effector memory (CD44 $\left.{ }^{\text {high }} \mathrm{CD} 62 \mathrm{~L}^{\text {low }}\right)$ but decreased the percentage of central memory $\left(\mathrm{CD} 44^{\text {high }} \mathrm{CD} 62 \mathrm{~L}^{\text {high }}\right) \mathrm{CD}^{+}$ and $\mathrm{CD}^{+} \mathrm{T}$ cells in the spleens. The percentage of the tumor infiltrating central and effector memory cells was not affected by parsaclisib (Supplemental Fig. 2C).

Parsaclisib Is Associated with Reduced Hepatotoxicity. We postulated that the liver toxicities observed with the first-generation $\mathrm{PI} 3 \mathrm{~K} \delta$ inhibitors idelalisib, duvelisib, and INCB040093 are caused by off-target effects that can be linked to this class of molecules. The purine motif is identical within all three compounds, and each contains a phenyl-substituted bicyclic aryl core (Table 1). Parsaclisib is derived from a different scaffold consisting of a pyrazolopyrimidine in place of the purine and a lactam-substituted monocycle core (Table 1 ).

In 28-day repeat dose GLP safety studies of parsaclisib, no evidence of direct hepatotoxicity was observed in dogs or rats; in 8- and 9-day non-GLP rat studies, evidence of hepatotoxicity was observed, but only at very large multiples ( $>25$-fold) of the exposures observed in patients administered a $20-\mathrm{mg}$, once-daily dose. Importantly, human clinical trials have also shown reduced clinically relevant, liver-related safety findings or adverse events (Forero-Torres et al., 2019) (Table 1), supporting our hypothesis that liver toxicities associated with first-generation PI3K $\delta$ inhibitors are the result of off-target effects and that alternative molecules with a reduced potential for hepatotoxicity can be developed.

Umbralisib (TGR-1202) is another next-generation PI3K $\delta$ inhibitor. Like parsaclisib, only a small proportion of patients experienced liver-related toxicities in clinical studies (Burris et al., 2018) (Table 1). The structure of umbralisib contains a phenyl-substituted bicyclic aryl core but replaces the purine substituent with a pyrazolopyrimidine similar to parsaclisib (Table 1). Thus, based on the structure analysis and the corresponding preclinical and clinical safety study findings, we hypothesize that the off-target liver toxicity associated with the three first-generation $\mathrm{PI} 3 \mathrm{~K} \delta$ inhibitors may be due to an untoward interaction involving the common purine substituent within these structures.

\section{Discussion}

We present the preclinical characterization of parsaclisib, a structurally novel $\mathrm{PI} 3 \mathrm{~K} \delta$ inhibitor, in clinical trials for the treatment of B-cell malignancies. The results demonstrate that parsaclisib is a highly potent and selective PI3K $\delta$ inhibitor in various biochemical and cell-based assays, with reduced hepatotoxicity compared with first-generation PI3K $\delta$ inhibitors (which has limited their utility in the clinical setting). The first-generation PI3K $\delta$ inhibitors share a common purine constituent in their chemical structures. Parsaclisib is the product of a discovery program aimed at investigating whether an alternative scaffold could circumvent the hepatotoxicity associated with first-generation PI3K $\delta$ inhibitors. Indeed, parsaclisib has demonstrated minimal hepatotoxicity in preclinical and clinical studies. Therefore, we hypothesize that the hepatotoxicity associated with the first-generation $\mathrm{PI} 3 \mathrm{~K} \delta$ inhibitors is structure-based rather than mechanism-based.

Consistent with the therapeutic potential of parsaclisib for hematologic malignancies, proliferation of MCL and DLBCL cell lines in vitro and tumor growth in MCL and DLBCL xenograft models in vivo were potently inhibited by parsaclisib. Parsaclisib inhibited in vitro proliferation of four MCL cell lines and the growth of 1 MCL cell line (Rec-1) in a xenograft model. Signaling studies confirmed that the effect of parsaclisib in these cells is through inhibition of PI3K-mediated pathways. In keeping with these preclinical results, a high ORR $(67 \%)$ was achieved among patients receiving parsaclisib for relapsed or refractory MCL in a recent phase $1 / 2$ study (Forero-Torres et al., 2019). MCL is genetically characterized by the $\mathrm{t}(11 ; 14)$ translocation that juxtaposes the protooncogene $C C N D 1$, which encodes cyclin D1 at chromosome 11 , to the $I G H$ gene, which encodes Ig heavy chain at chromosome 14. As a consequence, cyclin D1, which is not expressed in normal B cells, becomes constitutively overexpressed. This genetic alteration is thought to be the primary event in MCL pathogenesis, probably by facilitating cell cycle

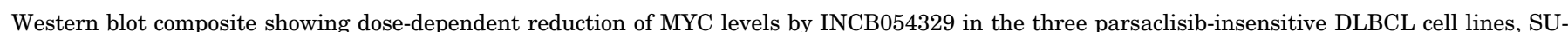

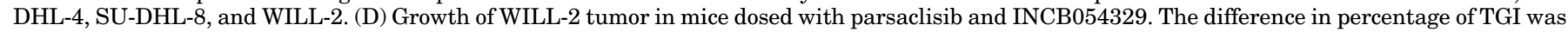

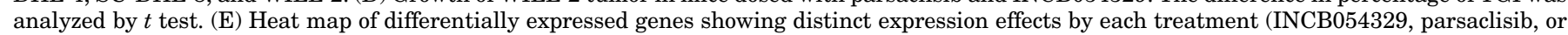

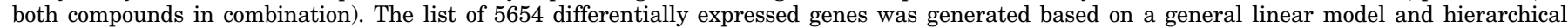

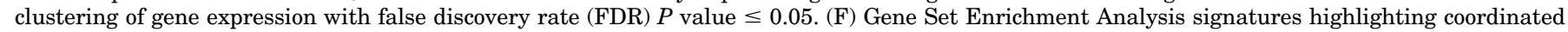

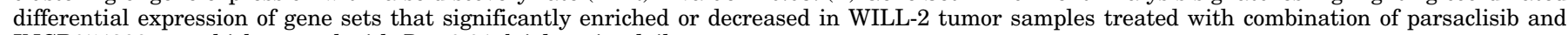
INCB054329 vs. vehicle control with $P<0.01$. b.i.d., twice daily. 
A

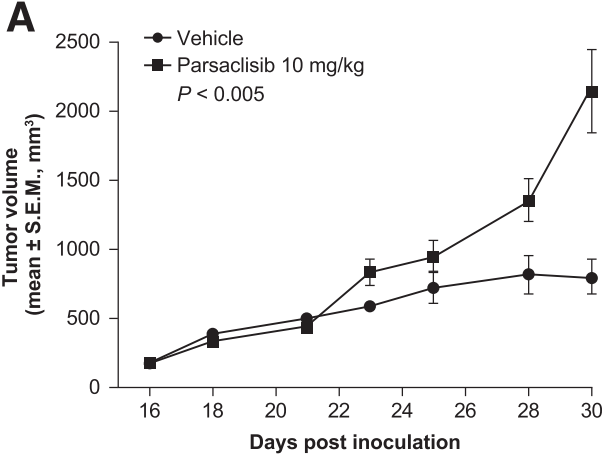

C

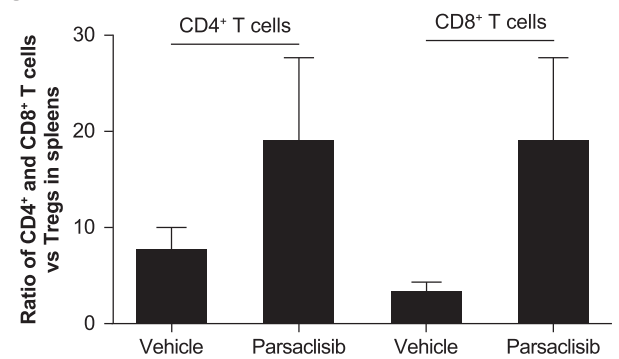

E

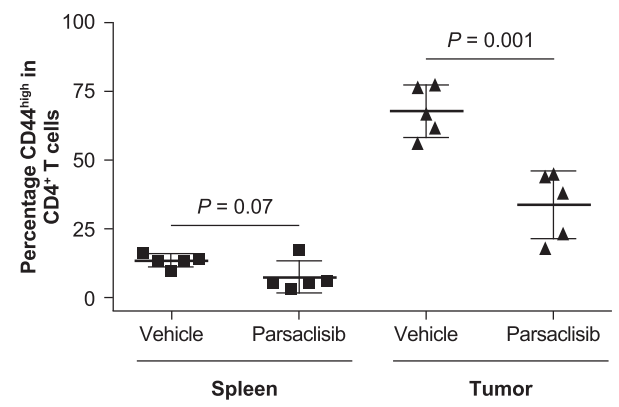

B

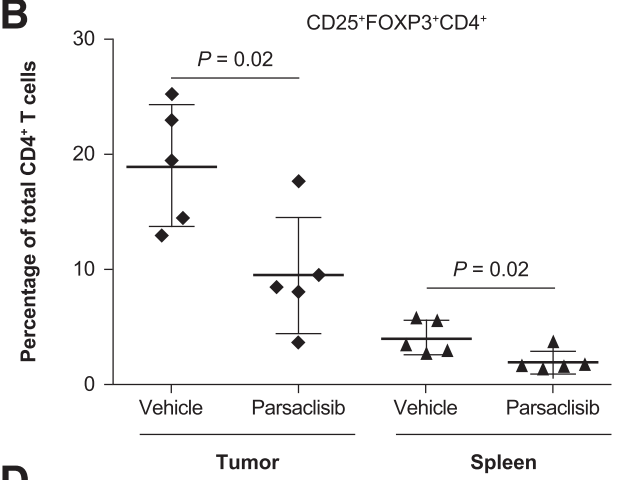

D

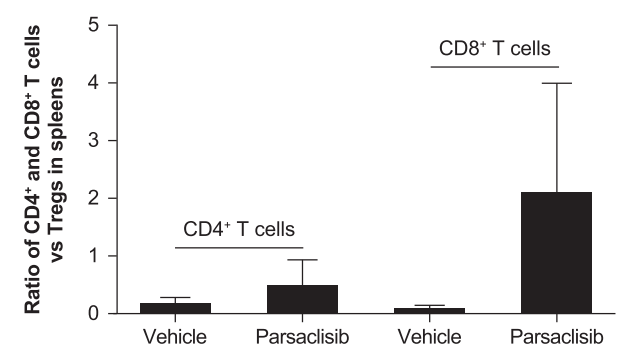

$\mathbf{F}$

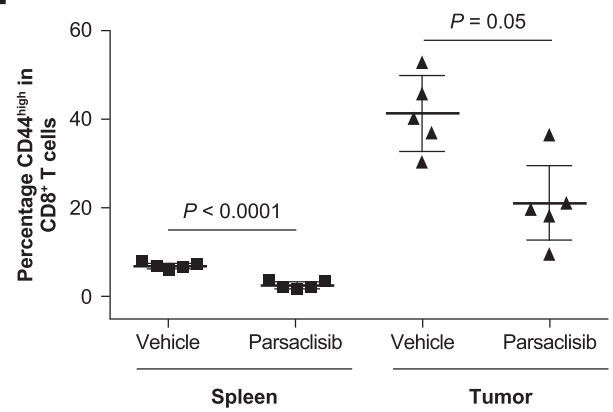

Fig. 5. Parsaclisib is efficacious in reducing tumor growth in the A20 model of lymphoma. (A) Growth of A20 tumor in female BALB/c mice with or without parsaclisib treatment. The treatment of the tumor-bearing mice started 7-24 days after tumor inoculation. (B) Effect of parsaclisib on the percentage of Tregs $\left(\mathrm{CD} 25^{+} \mathrm{FOXP}^{+} \mathrm{CD}^{+}\right)$in the $\mathrm{CD} 4^{+} \mathrm{T}$ cells isolated from spleens and tumors in the A20 model. Effect of parsaclisib on the ratio of $\mathrm{CD} 4^{+}$and $\mathrm{CD} 8^{+} \mathrm{T}$ cells vs. Tregs in spleens (C) and tumors (D) of the A20 model. Effect of parsaclisib on the percentage of memory/activated cells $\left(\mathrm{CD} 44^{+}\right)$ within the $\mathrm{CD}^{+}(\mathrm{E})$ and $\mathrm{CD}^{+}(\mathrm{F}) \mathrm{T}$-cell subpopulations in spleens and tumors of the A20 model. In (B-F), the mice were treated with parsaclisib at $10 \mathrm{mg} / \mathrm{kg}$ once daily or vehicle control for 7 days before being euthanized for collection and processing of tumors and spleens for fluorescence-activated cell sorting analysis. deregulation at the G1-S phase transition (Jares et al., 2007). However, the AKT survival pathway is activated in MCL, particularly in the blastoid variants with the loss of phosphatase and tensin homolog expression (Rudelius et al., 2006). The MCL cell lines tested in this study contain the $t(11 ; 14)$ translocation, yet they are highly sensitive to PI3K $\delta$ inhibition by parsaclisib, suggesting that the PI3K $\delta / A K T$ signaling pathway is as critical as cyclin D1 overexpression for the development and/or survival of MCL.

Evidence suggests that PI3K $\delta$ signaling may also play a role in DLBCL pathogenesis. For example, a single mutation in the catalytic domain of PI3K $\delta$ maintained the level of pAKT after cytokine withdrawal in recombinant cells (Zhang et al., 2013). Likewise, chronic BCR signaling, which activates $\mathrm{PI} 3 \mathrm{~K} \delta$, might be a pathogenic mechanism in the activated B-cell-like subtype of DLBCL (Davis et al., 2010). We found that not all DLBCL cell lines are sensitive to PI3K $\delta$ inhibition despite inhibition of the PI3K signaling pathway by parsaclisib. One similarity shared by the parsaclisib-sensitive DLBCL cell lines is the presence of the $\mathrm{t}(14 ; 18)$ chromosome translocation, which juxtaposes the $B C L 2$ gene on chromosome 18 to the $I G H$ enhancer on chromosome 14, resulting in BCL2 protein overexpression and inhibition of apoptosis (McDonnell et al., 1989). Our observations suggest that inhibition of the growth-/survival-promoting effects of PI3K signaling can override the antiapoptotic effect of BCL2 in these cells. Interestingly, the $t(14 ; 18)$ translocation frequency is about 15\%-30\% in DLBCL and 85\% in FL (Johnson et al., 2009), which is similar to the clinical response rate among patients with relapsed or refractory DLBCL (30\%) and FL $(71 \%)$ receiving parsaclisib (Forero-Torres et al., 2019).

The parsaclisib-insensitive cell lines tested all have exaggerated MYC expression, and MYC reduction by BET inhibitor treatment correlated closely with cell proliferation inhibition, indicating that $M Y C$ dysregulation may be a driver of proliferation in these cells. Indeed, parsaclisib combination with the BET inhibitor INCB054329 showed synergistic antitumor activity in vivo, demonstrating that insensitivity to PI3K $\delta$ inhibition by parsaclisib may be overcome by MYCtargeting strategies such as BET inhibition (Delmore et al., 2011; Boi et al., 2015). The enhanced efficacy observed with combined PI3K $\delta$ and BET inhibition likely reflects enrichment of a p53 pathway gene expression profile, as well as repression of MYC transcriptional targets. Previous studies have established a dual mechanism of action for the anti-B-cell malignancy effect of $\mathrm{PI} 3 \mathrm{~K} \delta$ inhibition: direct decrease of cell survival/proliferation as well as inhibition of chemokine production that aids retention of malignant B cells in protective tissue microenvironments. Clinically, PI3K $\delta$ inhibition has been shown to cause rapid lymph node 


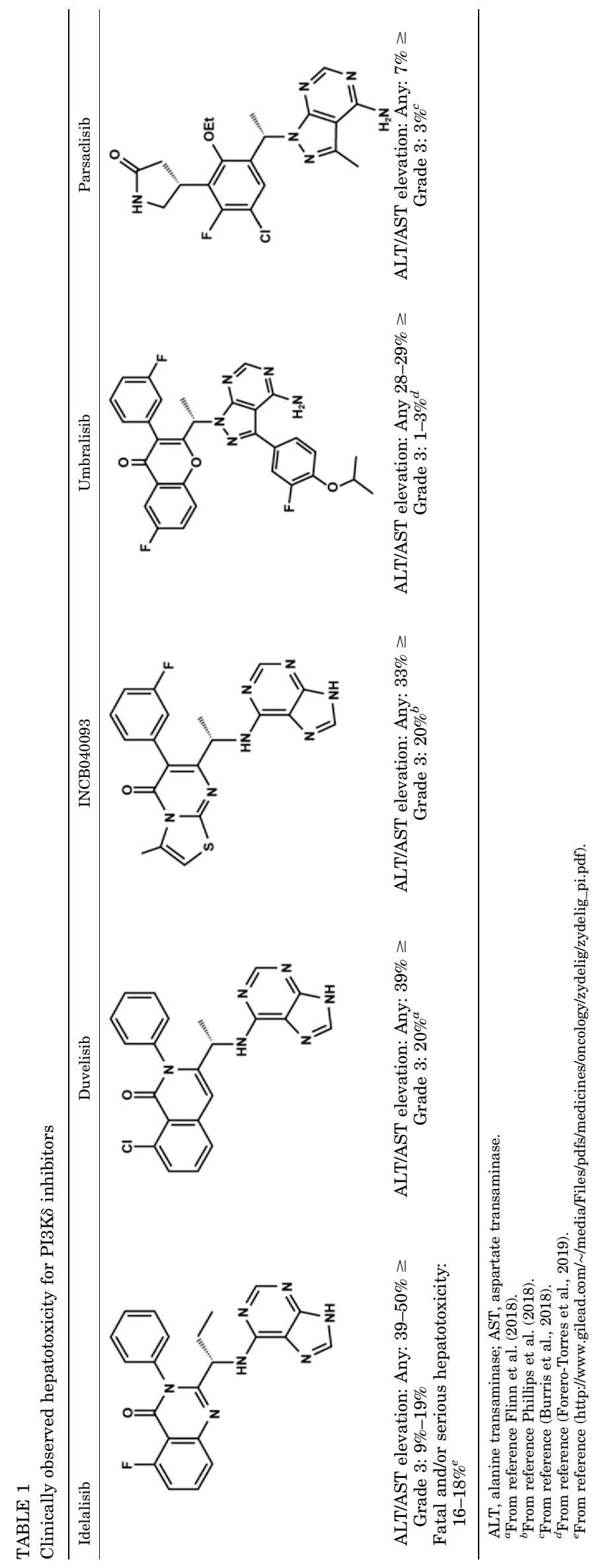

shrinkage and transient lymphocytosis in patients with CLL. It is believed that PI3K $\delta$ functions to promote tumor cell connection with stroma cells through an intricate chemokine and chemokine receptor network (Hoellenriegel et al., 2011). We found that the expression of multiple chemokines and chemokine receptors was substantially decreased upon combination inhibition. Combining PI3K $\delta$ with BET inhibition may be useful in the clinic for treating patients with DLBCL with high MYC expression levels with a suboptimal response to PI3K inhibition alone. This is based on the rationale that PI3K $\delta$ inhibition can break down the protective network of tumor cells by chemokine signaling, making the lymphoma more susceptible to direct growth/survival pathway inhibition by a BET inhibitor. The inhibitory effect of parsaclisib on the chemokines and chemokine receptors is consistent with the current hypothesis. An additional approach to MYC-PI3K $\delta$ axis inhibition in these resistant tumors could involve inhibition of proviral integration site for Moloney murine leukemia virus (PIM) kinases, which occupy key nodes in signaling networks that are highly interconnected with those mediated by PI3K $\delta$ (Forero-Torres et al., 2019). A previous study demonstrated additive or synergistic tumor-growth inhibitory effects of parsaclisib in combination with the panproviral integration site for Moloney murine leukemia virus kinase inhibitor, INCB053914 (Koblish et al., 2018), and a clinical investigation of this combination has been opened (NCT03688152).

Based on previous findings that $\mathrm{PI} 3 \mathrm{~K} \delta$ activity in different immune cells may have tumor protective functions (Ali et al., 2014), we explored whether parsaclisib could function as an immune modulator in the tumor microenvironment by using the murine A20 model of lymphoma. Although A20 cells were not sensitive to parsaclisib inhibition in vitro, tumor growth was suppressed in the murine A20 model, suggesting that part of the tumor inhibitory effect of parsaclisib in vivo may be through immune modulation. Indeed, parsaclisib reduced the number of Tregs in the tumors and spleens of treated mice. This result is consistent with recent clinical data showing that combined parsaclisib and pembrolizumab (an antiprogrammed cell death-1 antibody) significantly reduced the number of Tregs in patients with solid tumors (Kirkwood et al., 2018). Tregs suppress antitumor immune responses by inhibiting cytotoxic T-cell activity in the tumor microenvironment (Chaudhary and Elkord, 2016), and it is therefore conceivable that immune activation may occur after parsaclisib treatment. Interestingly, while preparing this report, a study was published supporting a critical role for PI3K $\delta$ in CLL pathogenesis through Tregs (Dong et al., 2019).

Memory $\mathrm{CD} 8^{+} \mathrm{T}$ cells are superior mediators of antitumor immunity compared with terminally differentiated effector cells because of their greater proliferative potential (Gattinoni et al., 2005), but conflicting data exist on the role of PI3K $\delta$ in the regulation of T-cell differentiation. A recent study suggests that PI3K $\delta$ inhibition could delay terminal differentiation of $\mathrm{CD}^{+} \mathrm{T}$ cells and maintain the memory phenotype and their ability to produce cytokines and granzyme B (Abu Eid et al., 2017). However, in $\mathrm{p} 110 \delta^{\mathrm{D} 910 \mathrm{~A} / \mathrm{D} 910 \mathrm{~A}}$ mice, which lack functional PI3K $\delta$ catalytic activity, the expression of CD44 (a marker of memory T cells), was considerably reduced on $\mathrm{CD} 4^{+}$ and $\mathrm{CD} 8^{+} \mathrm{T}$ cells (Okkenhaug et al., 2002). Similarly, PI3K $\delta$ inactivation reduced the number of $\mathrm{CD} 44^{\text {high }} \mathrm{CD}^{+}$and $\mathrm{CD} 44^{\text {high }} \mathrm{CD} 4^{+} \mathrm{T}$ cells in the lymph nodes of mice bearing 
4T1 or EL3 tumors (Ali et al., 2014). In the lymphoma A20 model, parsaclisib treatment significantly reduced memory CD4 and CD8 T cells (CD44 ${ }^{\text {high }}$ ) in the spleen and tumor, making it unlikely that $\mathrm{T}$-cell differentiation plays a role in the mechanism of the observed antitumor activity of parsaclisib.

In summary, parsaclisib is a novel, highly potent, and selective PI3K $\delta$ inhibitor that has advanced into phase 2 clinical trials enrolling patients with hematologic B-cell malignancies. Preclinical validation has shown that parsaclisib impedes B-cell lymphoma cell line growth in vitro and in vivo. Parsaclisib also regulates the functions of $\mathrm{B}$ and $\mathrm{T}$ cells, theoretically making the tumor microenvironment less conducive to tumor growth. Additionally, insensitivity to parsaclisib in DLBCL due to aberrant MYC overexpression may be overcome by combination with a BET inhibitor. Importantly, parsaclisib demonstrated reduced hepatotoxicity in preclinical and clinical studies when compared with the first-generation compounds. Parsaclisib is also distinct from other next-generation agents (e.g., umbralisib with a daily dose of $800 \mathrm{mg}$ ) in being administered as a $20-\mathrm{mg}$, once-daily dosing regimen, which has been shown to completely inhibit signaling through the PI $3 \mathrm{~K} \delta$ pathway. Our results provide the mechanistic basis to fully support the ongoing efforts in the development of parsaclisib for treating patients with B-cell malignancies. The potential immunomodulatory activity observed in these preclinical studies implies that parsaclisib could have a broader application as a therapy outside of hematologic oncology.

\section{Acknowledgments}

We would like to acknowledge Jordan Fridman and Kris Vaddi for their suggestions during the characterization of parsaclisib. With this manuscript, we remember the contributions of our colleague Y.-L.L. Medical writing assistance was provided by Abigail Marmont, CMPP, of Envision Pharma Group (Philadelphia, PA) and funded by Incyte Corporation.

\section{Authorship Contributions}

Participated in research design: Shin, Stubbs, Koblish, Yue, Soloviev, Gao, Diamond, Shuey, Yeleswaram, Combs, Newton, Scherle.

Conducted experiments: Shin, Soloviev, K. H. Wang, Q. Wang, Gao, Feldman, Yang, Hall, Hansbury, O'Connor, Leffet, Collins, Katiyar, He, Waeltz, Collier.

Contributed new reagents or analytic tools: Yue, Douty, Metcalf, Yao, Combs.

Performed data analysis: Shin, Stubbs, Koblish, Soloviev, Y.-L. Li, Y. Li, Liu, Burn, Covington, Diamond, Shuey, Roberts, Yeleswaram, Hollis, Metcalf, Yao, Huber, Combs, Newton, Scherle.

Wrote or contributed to the writing of the manuscript: Shin, Stubbs, Koblish, Shuey, Combs, Newton, Scherle.

\section{References}

Abu Eid R, Ahmad S, Lin Y, Webb M, Berrong Z, Shrimali R, Kumai T, Ananth S, Rodriguez PC, Celis E, et al. (2017) Enhanced therapeutic efficacy and memory of tumor-specific CD8 T cells by ex vivo PI3K- $\delta$ inhibition. Cancer Res 77:4135-4145.

Ali K, Soond DR, Pineiro R, Hagemann T, Pearce W, Lim EL, Bouabe H, Scudamore CL, Hancox T, Maecker H, et al. (2014) Inactivation of PI(3)K p110 breaks regulatory T-cell-mediated immune tolerance to cancer. Nature 510:407-411.

Boi M, Gaudio E, Bonetti P, Kwee I, Bernasconi E, Tarantelli C, Rinaldi A, Testoni M, Cascione L, Ponzoni M, et al. (2015) The BET bromodomain inhibitor OTX015 affects pathogenetic pathways in preclinical B-cell tumor models and synergizes with targeted drugs. Clin Cancer Res 21:1628-1638.

Burris HA III, Flinn IW, Patel MR, Fenske TS, Deng C, Brander DM, Gutierrez M,

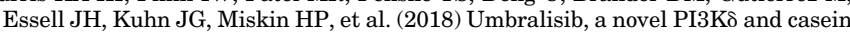
kinase- $1 \varepsilon$ inhibitor, in relapsed or refractory chronic lymphocytic leukaemia and lymphoma: an open-label, phase 1, dose-escalation, first-in-human study. Lancet Oncol 19:486-496.
Chaudhary B and Elkord E (2016) Regulatory T cells in the tumor microenvironment and cancer progression: role and therapeutic targeting. Vaccines (Basel) 4:E28.

Ciraolo E, Morello F, Hobbs RM, Wolf F, Marone R, Iezzi M, Lu X, Mengozzi G, Altruda F, Sorba G, et al. (2010) Essential role of the p110beta subunit of phosphoinositide 3-OH kinase in male fertility. Mol Biol Cell 21:704-711.

Coutré SE, Barrientos JC, Brown JR, de Vos S, Furman RR, Keating MJ, Li D O’Brien SM, Pagel JM, Poleski MH, et al. (2015) Management of adverse events associated with idelalisib treatment: expert panel opinion. Leuk Lymphoma 56: 2779-2786.

Davis RE, Ngo VN, Lenz G, Tolar P, Young RM, Romesser PB, Kohlhammer H, Lamy L, Zhao H, Yang Y, et al. (2010) Chronic active B-cell-receptor signalling in diffuse large B-cell lymphoma. Nature 463:88-92.

Delmore JE, Issa GC, Lemieux ME, Rahl PB, Shi J, Jacobs HM, Kastritis E, Gilpatrick T, Paranal RM, Qi J, et al. (2011) BET bromodomain inhibition as a therapeutic strategy to target c-Myc. Cell 146:904-917.

Dong S, Harrington BK, Hu EY, Greene JT, Lehman AM, Tran M, Wasmuth RL, Long M, Muthusamy N, Brown JR, et al. (2019) PI3K p1108 inactivation antagonizes chronic lymphocytic leukemia and reverses $\mathrm{T}$ cell immune suppression. $J$ Clin Invest 129:122-136.

Evans CA, Liu T, Lescarbeau A, Nair SJ, Grenier L, Pradeilles JA, Glenadel Q, Tibbitts T, Rowley AM, DiNitto JP, et al. (2016) Discovery of a selective phosphoinositide-3-kinase (PI3K)- $\gamma$ inhibitor (IPI-549) as an immuno-oncology clinical candidate. ACS Med Chem Lett 7:862-867.

Fabre S, Lang V, Harriague J, Jobart A, Unterman TG, Trautmann A, and Bismuth G (2005) Stable activation of phosphatidylinositol 3-kinase in the T cell immunological synapse stimulates Akt signaling to FoxO1 nuclear exclusion and cell growth control. J Immunol 174:4161-4171.

Flinn IW, Patel M, Oki Y, Horwitz S, Foss FF, Allen K, Douglas M, Stern H, Sweeney $J$, Kharidia J, et al. (2018) Duvelisib, an oral dual PI3K- $\delta$, $\gamma$ inhibitor, shows clinical activity in indolent non-Hodgkin lymphoma in a phase 1 study. Am J Hematol 93:1311-1317.

Forero-Torres A, Ramchandren R, Yacoub A, Wertheim MS, Edenfield WJ, Caimi P Gutierrez M, Akard L, Escobar C, Call J, et al. (2019) Parsaclisib, a potent and highly selective PI3K $\delta$ inhibitor, in patients with relapsed or refractory B-cell malignancies. Blood 133:1742-1752.

Gattinoni L, Klebanoff CA, Palmer DC, Wrzesinski C, Kerstann K, Yu Z, Finkelstein SE, Theoret MR, Rosenberg SA, and Restifo NP (2005) Acquisition of full effector function in vitro paradoxically impairs the in vivo antitumor efficacy of adoptively transferred CD8+ T cells. J Clin Invest 115:1616-1626.

Greenwell IB, Flowers CR, Blum KA, and Cohen JB (2017) Clinical use of PI3K inhibitors in B-cell lymphoid malignancies: today and tomorrow. Expert Rev Anticancer Ther 17:271-279.

Haylock-Jacobs S, Comerford I, Bunting M, Kara E, Townley S, Klingler-Hoffmann M, Vanhaesebroeck B, Puri KD, and McColl SR (2011) PI3Kס drives the pathogenesis of experimental autoimmune encephalomyelitis by inhibiting effector T cell apoptosis and promoting Th17 differentiation. J Autoimmun 36: $278-287$

Hers I (2007) Insulin-like growth factor-1 potentiates platelet activation via the IRS/ PI3Kalpha pathway. Blood 110:4243-4252.

Hoellenriegel J, Meadows SA, Sivina M, Wierda WG, Kantarjian H, Keating MJ, Giese N, O'Brien S, Yu A, Miller LL, et al. (2011) The phosphoinositide 3'-kinase delta inhibitor, CAL-101, inhibits B-cell receptor signaling and chemokine networks in chronic lymphocytic leukemia. Blood 118:3603-3612.

Ilic N, Utermark T, Widlund HR, and Roberts TM (2011) PI3K-targeted therapy can be evaded by gene amplification along the MYC-eukaryotic translation initiation factor 4E (eIF4E) axis. Proc Natl Acad Sci USA 108:E699-E708.

Jares P, Colomer D, and Campo E (2007) Genetic and molecular pathogenesis of mantle cell lymphoma: perspectives for new targeted therapeutics. Nat Rev Cancer 7:750-762.

Johnson NA, Savage KJ, Ludkovski O, Ben-Neriah S, Woods R, Steidl C, Dyer MJ, Siebert R, Kuruvilla J, Klasa R, et al. (2009) Lymphomas with concurrent BCL2 and MYC translocations: the critical factors associated with survival. Blood 114: $2273-2279$

Kirkwood JM, Lannotti N, Cho D, O'Day S, Gibney G, Hodi S, Munster P, Hoyle P,

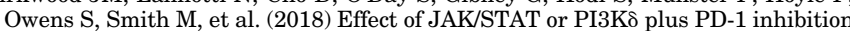
on the tumor microenvironment: biomarker results from a phase $1 \mathrm{~b}$ study in patients with advanced solid tumors, in Proceedings of the 109th Annual Meeting of the American Association for Cancer Research; 2018 April 14-18; Chicago, IL. pp ACT176.

Koblish H, Li YL, Shin N, Hall L, Wang Q, Wang K, Covington M, Marando C, Bowman K, Boer J, et al. (2018) Preclinical characterization of INCB053914, a novel pan-PIM kinase inhibitor, alone and in combination with anticancer agents, in models of hematologic malignancies. PLoS One 13:e0199108.

Kurebayashi Y, Nagai S, Ikejiri A, Ohtani M, Ichiyama K, Baba Y, Yamada T, Egami S, Hoshii T, Hirao A, et al. (2012) PI3K-Akt-mTORC1-S6K1/2 axis controls Th17 differentiation by regulating Gfi1 expression and nuclear translocation of ROR $\gamma$. Cell Rep 1:360-373.

Lampson BL, Kasar SN, Matos TR, Morgan EA, Rassenti L, Davids MS, Fisher DC, Freedman AS, Jacobson CA, Armand P, et al. (2016) Idelalisib given front-line for treatment of chronic lymphocytic leukemia causes frequent immune-mediated hepatotoxicity. Blood 128:195-203.

Lannutti BJ, Meadows SA, Herman SE, Kashishian A, Steiner B, Johnson AJ, Byrd JC, Tyner JW, Loriaux MM, Deininger M, et al. (2011) CAL-101, a p110 selective phosphatidylinositol-3-kinase inhibitor for the treatment of B-cell malignancies, inhibits PI3K signaling and cellular viability. Blood 117:591-594.

Liu P, Cheng H, Santiago S, Raeder M, Zhang F, Isabella A, Yang J, Semaan DJ, Chen C, Fox EA, et al. (2011) Oncogenic PIK3CA-driven mammary tumors frequently recur via PI3K pathway-dependent and PI3K pathway-independent mechanisms. Nat Med 17:1116-1120. 
222

Shin et al.

McDonnell TJ, Deane N, Phat FM, Nunez G, Jaeger U, McKearn JP, and Korsmeyer SJ (1989) bcl-2-immunoglobulin transgenic mice demonstrate extended B cell survival and follicular lymphoproliferation. Cell 57:79-88.

Okkenhaug K, Bilancio A, Farjot G, Priddle H, Sancho S, Peskett E, Peace W, Meek $\mathrm{SE}$, Salpekar A, Waterfield MD, et al. (2002) Impaired B and T cell antigen receptor signaling in p1108 PI 3-kinase mutant mice. Science 297:1031-1034.

Okkenhaug K, Patton DT, Bilancio A, Garçon F, Rowan WC, and Vanhaesebroeck B (2006) The p1108 isoform of phosphoinositide 3-kinase controls clonal expansion and differentiation of Th cells. J Immunol 177:5122-5128.

Patton DT, Garden OA, Peace WP, Clough LE, Monk CR, Lung E, Rowan WC, Sancho S, Walker LS, Vanhaesebroeck B, et al. (2006) Cutting edge: the posphoinositide 3-kinase p110 $\delta$ is critical for the function of $\mathrm{CD} 4^{+} \mathrm{CD} 25+\mathrm{Foxp}^{+}$ regulatory T cells. J Immunol 177:6598-6602.

Phillips TJ, Forero-Torres A, Sher T, Diefenbach CS, Johnston P, Talpaz M, Pulini J, Chou L, Scherle P, Chen X, et al. (2018) Phase 1 study of the PI3Kס inhibitor INCB040093 \pm JAK1 inhibitor itacitinib in relapsed/refractory B-cell lymphoma. Blood 132:293-306.

Puri KD and Gold MR (2012) Selective inhibitors of phosphoinositide 3-kinase delta: modulators of B-cell function with potential for treating autoimmune inflammatory diseases and B-cell malignancies. Front Immunol 3:256.

Richardson AI, Zhang D, Woodroof J, and Gui W (2019) p53 expression in large B-cell lymphomas with MYC extra copies and CD99 expression in large B-cell lymphomas in relation to MYC status. Hum Pathol 86:21-31.

Roller A, Perino A, Dapavo P, Sorn E, Okkenhaug K, Kirsch E, and Ii H (2012) Blockade of phosphatidylinositol 3-kinase PI3K $\delta$ or PI 3K $\gamma$ reduces IL-17 and ameliorates imiquimod-induced psoriasis-like dermatitis. $J$ Immunol 189: 4612-4620.

Rudelius M, Pittaluga S, Nishizuka S, Phat TH, Fend F, Jaffe ES, QuintanillaMartinez L, and Raffeld M (2006) Constitutive activation of Alt contributes to the pathogenesis and survival of mantle cell lymphoma. Blood 108:1668-1676.

Schrader A, Bentink S, Spang R, Lenze D, Hummel M, Ko M, Arrand JR, Murray PG, Trümper L, Kube D, et al. (2012) High Myc activity is an independent negative prognostic factor for diffuse large B cell lymphomas. Int J Cancer 131:E348-E361.
Shin N, Li YL, Mi S, Wang KH, Hall L, Katiyar K, Wang Q, Yang G, Rumberger B, Leffet L, et al. (2018) INCB040093 is a novel PI3Kס inhibitor for the treatment of B cell lymphoid malignancies. J Pharmacol Exp Cher 364:120-130.

Sound DR, Bjørgo E, Moltu K, Dale VQ, Patton DT, Torgersen KM, Galloway F, Twomey B, Clark J, Gaston JS, et al. (2010) PI3K p110ઠ regulates T-cell cytokine production during primary and secondary immune responses in mice and humans. Blood 115:2203-2213.

Steinbach EC, Kobayashi T, Russo SM, Sheikh SZ, Gipson GR, Kennedy ST, Uno JK, Mishima Y, Borst LB, Lu B, et al. (2014) Innate PI3K p110 regulates Th1/Th17 development and microbiota-dependent colitis. J Immunol 192:3958-3968.

Stubs MC, Burn TC, Sparks R, Maduskuie T, Diamond S, Rupar M, Wen X, Volgina A, Zolotarjova N, Waeltz P, et al. (2019) The novel bromodomain and extratermina domain inhibitor INCB054329 induces vulnerabilities in myeloma cells that inform rational combination strategies. Chin Cancer Res 25:300-311.

Wee S, Wiederschain D, Maira SM, Loo A, Miller C, deBeaumont R, Stegmeier F, Yo YM, and Lengauer C (2008) PTEN-deficient cancers depend on PIK3CB. Proc Natl Accad Sci USA 105:13057-13062.

Whyte DB and Holbeck SL (2006) Correlation of PIK3Ca mutations with gene expression and drug sensitivity in NCI-60 cell lines. Biochem Biophys Res Common 340:469-475.

Ye EW, Li Y-L, Duty B, He C, Mi S, Wayland B, Maduskuie T, Falahatpisheh N, Sparks RB, Polam P, et al. (2019) INCB050465 (parsaclisib), a novel nextgeneration inhibitor of phosphoinositide 3-kinase delta (PI3Kס). ACS Med Chem Lett 10:1554-1560.

Chang J, Grubor V, Love CL, Banerjee A, Richards KL, Mieczkowski PA, Dunphy C, Chi W, Au WY, Srivastava G, et al. (2013) Genetic heterogeneity of diffuse large B-cell lymphoma. Proc Natl Acid Sci USA 110:1398-1403.

Address correspondence to: Nu Shin, Incyte Research Institute, Incyte Corporation, 1801 Augustine Cut Off, Wilmington, DE 19803. E-mail: nshin@ incyte.com 